\title{
The Utility of the Novel Hematologic Parameters for Predicting in Hospital Mortality in Patients with Acute Mesenteric Ischemia
}

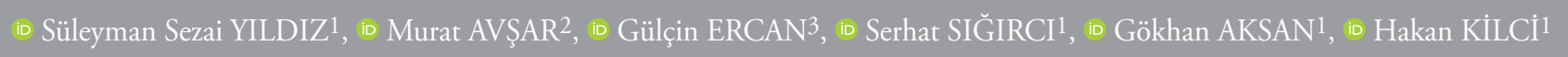

1University of Health Sciences, Şişli Hamidiye Etfal Training and Research Hospital, Clinic of Cardiology, İstanbul, Turkey

2University of Health Sciences, Okmeydanı Training and Research Hospital, Clinic of Cardiology, İstanbul, Turkey

${ }_{3}$ University of Health Sciences, Bağcllar Training and Research Hospital, Clinic of General Surgery, İstanbul, Turkey

\begin{abstract}
Objective: Acute mesenteric ischemia (AMI) is recognized as a vascular emergency, having high mortality and requiring rapid and efficient and treatment. In this study, we aimed to assess the levels of neutrophil to lymphocyte ratio (NLR) and platelet to lymphocyte ratio (PLR) in patients with AMI and to search for a relationship between the NLR and PLR values and inhospital mortality of patients with AMI.

Methods: In total, one hundred and twenty three patients (67 AMI+ and 56 AMI-) were included in this retrospective study. Sixty seven patients diagnosed as having AMI following computed tomography angiography after presenting to the emergency room were included in AMI (+) group. "Receiver Operating Characteristic" analysis was done to determine the optimal NLR and PLR thresholds in diagnosis and inhospital mortality of AMI. Also, univariate and multivariate analyses were made to evaluate the complete blood count parameters affecting the outcomes of patients with AMI.

Results: It was found that AMI+ patients had higher neutrophil count than AMI- patients, while the lymphocyte count was significantly lower $(\mathrm{p}<0.001 \mathrm{p}<0.001$, respectively). The NLR and PLR values were significantly higher in AMI+patients than in AMI-patients $(\mathrm{p}<0.001, \mathrm{p}<0.001$, respectively). In the multiple logistic regression analysis, only NLR and red cell distribution width were identified as independent predictors of inhospital mortality of patients with AMI [odds ratio $(\mathrm{OR})=1.171,95 \%$ confidence interval $(\mathrm{CI})=1.020-1.345, \mathrm{p}=0.026$; OR $=1.474$, 95\% $\mathrm{CI}=1.028-2.113, \mathrm{p}=0.035$, respectively]. Although PLR failed to predict inhospital mortality in AMI+ subjects (area under the curve (AUC $=0.597,95 \% \mathrm{CI}=0.459-0.734, \mathrm{p}<0.178$ ), NLR level greater than 10.1, measured on admission, predicted inhospital mortality in AMI+ subjects ( $\mathrm{AUC}=0.698,95 \% \mathrm{CI}=0.570-0.827, \mathrm{p}<0.006$ ).
\end{abstract}

Conclusion: Neutrophil-to-lymphocyte ratio is a reliable predictive marker in hospital mortality in patients with AMI.

Keywords: Neutrophil to lymphocyte ratio, acute mesenteric ischemia, mortality

Address for Correspondence: Süleyman Sezai YILDIZ, University of Health Sciences, Şişli Hamidiye Etfal Training and Research Hospital, Clinic of Cardiology, İstanbul, Turkey E-mail: sezai04@yahoo.com ORCID ID: orcid.org/0000-0003-2307-3504

Received: 13.09 .2018

Cite this article as: Yıldız SS, Avşar M, Ercan G, Sığırcı S, Aksan G, Kilci H. The Utility of the Novel Hematologic Parameters for Predicting in Hospital Mortality in Patients with Acute Mesenteric İschemia. Bezmialem Science 2019;7(4):259-64. 


\section{Introduction}

Although acute mesenteric ischemia (AMI) accounts only $1 \%$ of all hospitalizations with acute abdomen, this rate may increase up to $10 \%$ in patients over 70 years of age (1). Primary AMI is a vascular disease and can be classified by etiologies as follows: Mesenteric arterial embolism, mesenteric arterial thrombosis, mesenteric venous thrombosis, and non-occlusive mesenteric arterial ischemia (2). Predisposing risk factors are as follows: Atrial fibrillation, heart failure, coronary heart disease, arterial hypertension, severe valvular cardiac disease and peripheral arterial occlusion (3). In contrast with its low incidence, mesenteric ischemia may be associated with very high mortality rates up to $40-70 \%$ (4). Major factors responsible for high mortality include late presentation, absence of simple and reliable biochemical parameters and experienced radiologists, and late diagnosis (5). In contrast with other conditions with abdominal pain of acute onset, AMI is more likely to result in the development of irreversible complications (6). Early diagnosis is of utmost importance for reducing the mortality rate in AMI. Although computed tomography (CT) angiography is considered the gold standard imaging modality in early diagnosis, currently no standard biochemical parameters exist that can be used for routine diagnosis of this condition. Laboratory investigations frequently demonstrate leukocytosis, metabolic acidosis, an elevated D-dimer and elevated serum lactate (7-9). Laboratory studies are non-specific; while abnormal laboratory values may be helpful in bolstering suspicion for AMI, normal laboratory values do not exclude AMI.

Detection of embolic or thrombotic arterial occlusion of the superior mesenteric artery (SMA) in 70 to $80 \%$ of patients diagnosed as having AMI after presentation at emergency room has fueled an interest in the use of novel hematological parameters in complete blood count $(\mathrm{CBC})$ including NLR and PLR that indicate the inflammatory and thrombotic status (4,10-13). Although several studies suggested that high NLR and platelet to lymphocyte (PLR) may represent significant markers for early diagnosis of AMI, currently no data exists on the use of NLR and PLR in predicting inhospital mortality in AMI. The aim of this article is to search: (i) whether there exists an association between novel hematologic parameters (NLR, PLR) and AMI; (ii) whether elevated NLR and PLR counts are associated with mortality following AMI.

\section{Methods}

\section{Study Design and Setting}

In this retrospective study investigating novel $\mathrm{CBC}$ parameters that may have a predictive value in AMI mortality, a total of 67 patients diagnosed as having AMI following CT angiography after presenting to the emergency unit of our institute between 2010 and 2014 were included in AMI (+) group. The study was approved by the Local Ethics Committee.

\section{Selection of Participants}

A total of 56 age- and gender-matched individuals presenting with abdominal pain but without detection of AMI in CT angiography comprised the AMI (-) group. Patients with active infection, systemic inflammatory conditions, malignancy, advanced hepatic failure, renal failure, thyroid disease, use of corticosteroid or anti-inflammatory medications, and hematological conditions were excluded.

\section{Baseline Measurements}

Prior to the initiation of any medical treatments, venous blood samples were drawn from the antecubital vein and were collected in ethylene diamine tetraacetic acid containing tubes. Automatic blood counter (CELL-DYN Ruby, Abbott Diagnostics, Abbott Park, IL, USA) was used for whole blood count. NLR and PLR were calculated as the ratio of the neutrophils and platelets to lymphocytes, respectively, both obtained from the same automated blood samples that were taken at the time of admission to the study. CRP was measured using Cobas c501 analyzer (Roche Diagnostics, Indianapolis, IN).

\section{Statistical Analysis}

Descriptive statistics included mean, standard deviation, minimum, maximum, and median, while categorical variables were expressed as number and percent. Independent group comparisons were performed using Student t-test for variables with normal distribution and using Mann-Whitney $U$ test in the absence of normal distribution. Percent comparisons between independent groups were performed with chi-square analysis. Risk factors were analyzed using the logistic regression analysis. Receiver operating curve (ROC) curve analysis was used to detect the predictive value of PLR and NLR for AMI mortality. The sensitivity, specificity, and positive and negative predictive value (PPV and NPV) were calculated using different cut-off values. $\mathrm{P}$ values less than 0.05 were considered significant, and the confidence interval (CI) was $95 \%$. The statistical analyses were performed using SPSS, version 17 (SPSS, Chicago, Illinois, USA).

\section{Results}

Our study included a total of 123 subjects, 67 AMI (+) and 56 AMI (-) patients. All participants presented to the emergency room with abdominal pain. The baseline demographic characteristics and laboratory parameters of patients are shown in Table 1. There were no significant differences between the two groups in terms of age, gender, hemoglobin, platelet, and red cell distribution width (RDW). AMI+ patients had higher neutrophil count than AMI- patients, while the lymphocyte count was significantly lower $(p<0.001$ ve $p<0.001$, respectively). NLR was significantly higher in AMI+ patients than in AMIpatients $(\mathrm{p}<0.001)$ (Figure 1a). Platelet levels were not found to be significantly different between the groups $(\mathrm{p}=0.111)$. However, PLR was statistically higher in AMI+ subjects than 
in AMI- subjects $(\mathrm{p}<0.001)$ (Figure $1 \mathrm{~b})$. There was positive correlation between NLR and CRP ( $\mathrm{r}=0.544, \mathrm{p}<0.001)$, and between PLR and CRP $(r=0.525, \mathrm{p}<0.001)$.

Of AMI+ patients, $43 \%$ had atrial fibrilation (AF) rhythm. A lesion with $\geq 70 \%$ narrowing was present in superior mesenteric artery (SMA) and inferior mesenteric artery (IMA) in 88\% and $12 \%$ of the patients, respectively. The inhospital mortality rate was $48 \%$. Atrial fibrillation and mortality rates did not differ significantly between patients with SMA or IMA. Univariate logistic regression analysis showed significantly higher mean PLT and PLR values in patients with AF than in patients with sinus rhythm among those with $\mathrm{AMI}+(\mathrm{p}<0.001, \mathrm{p}=0.010$, respectively). In the multivariate logistic regression model

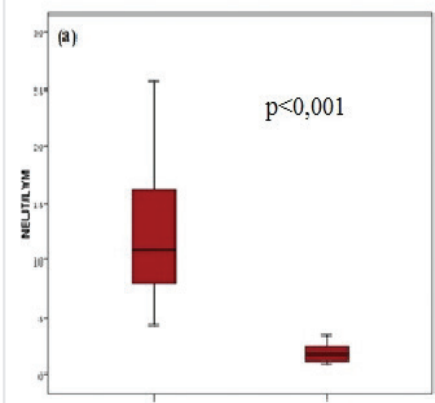

$\mathrm{AMI}+$

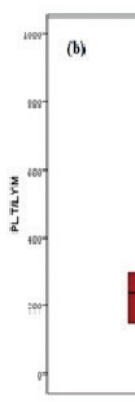

$\mathrm{AMI}+$
NLR and RDW emerged as the most significant factors [odds ratio $(\mathrm{OR})=1.171,95 \%$ confidence interval $(\mathrm{CI})=0.020$ 1.345, $\mathrm{p}=0.026, \mathrm{OR}=1.474,95 \% \mathrm{CI}=1.028-2.113, \mathrm{p}=0.035$, respectively) that predict mortality.

For determination of the best "cut-off" values of NLR and PLR in predicting the inhospital mortality among $\mathrm{AMI}+$ patients, a receiver operating curve (ROC) characteristics analysis was performed. The analysis of PLR failed to determine such cut-off values for predicting inhospital mortality (AUC value of 0.597 , 95\% CI=0.459-0.734, $\mathrm{p}<0.178)$. A NLR level greater than 10.1 , measured on admission, yielded an area under the curve (AUC) value of 0.698 (95\% CI=0.570-0.827, sensitivity $78 \%$, specificity $61 \%, \mathrm{p}<0.006$ ) (Figure $2 \mathrm{a}, 2 \mathrm{~b}$ ).
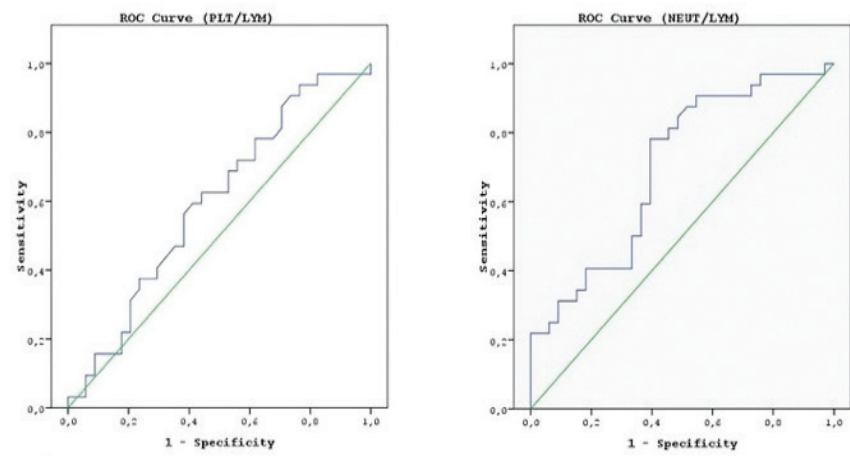

Figure 2. Comparison of receiver-operating characteristic analysis of NLR and PLR in prediction of AMI mortality. AMI; acute mesenteric ischemia, NLR; neutrophil/lymphocyte ratio, PLR; platelet/lymphocyte ratio
Figure 1. NLR and PLR values in diagnosis of AMI. NLR values in AMI + and AMI - groups (a), PLR values in AMI + and AMI group (b), AMl; acute mesenteric ischemia, NLR; neutrophil/ lymphocyte ratio, PLR; platelet/lymphocyte ratio
Table 1. Demographic and clinical characteristics of study population

Variable
Age (mean $\pm \mathrm{SD}$ )
Men, $\mathrm{n}(\%)$
Atrial fibrillation, $\mathrm{n}(\%)$
Diabetes mellitus, $\mathrm{n}(\%)$
Hypertension, $\mathrm{n}(\%)$
Coronary artery disease, $\mathrm{n}(\%)$
Cerebrovascular disease, $\mathrm{n}(\%)$
White blood cell count, $\times 10^{\%} / \mathrm{L}$
Neutrophils, $\times 10^{\%} / \mathrm{L}$
Lymphocytes (4L)
Hemoglobin ( $\mathrm{g} / \mathrm{dL}$ )
Platelet count, $\times 10^{3} \mathrm{HL}$
Red cell distribution width $(\%)$
Mean platelet volume $(\mathrm{fL})$
Neutrophil/lymphocyte ratio
Platelet/lymphocyte ratio

AMI $(n=67)$
$67 \pm 16$
$28(42)$
$29(43)$
$39(58)$
$55(82)$
$40(59)$
$12(18)$
$16.3 \pm 3.5$
$13.8 \pm 3.4$
$2.8 \pm 1.2$
$12.9 \pm 2.4$
$300 \pm 110$
$17.2 \pm 16.5$
$8.9 \pm 1.7$
$12.5 \pm 5.8$
$269 \pm 189$

Control $(n=56)$

$62 \pm 9$

$\mathrm{p}$ value

$24(43)$

0.61

$22(40)$

0.92

31 (56)

0.86

45 (80)

0.27

$34(60)$

0.21

9 (16)

0.56

0.8

$7.7 \pm 1.9$

$<0.001$

$4.7 \pm 1.5$

$<0.001$

$2.6 \pm 0.6$

$<0.001$

$13.2 \pm 2.4$

0.82

$259 \pm 61$

0.111

$16 \pm 14.3$

0.415

$7.5 \pm 1.9$

$<0.001$

$1.9 \pm 0.8$

$<0.001$

$99 \pm 20.6$

$<0.001$ 


\section{Discussion}

Our study showed that NLR and PLR levels increased in AMI+ patients. In addition, increased NLR levels, not PLR, predicted inhospital mortality in patients with AMI.

Despite the advances in diagnostic and imaging modalities, mortality rates in AMI remain high, partly due to the failure to consider AMI in differential diagnosis, use of unnecessary diagnostic procedures leading to diagnostic delay, as well as due to the fact that at the time AMI is diagnosed, it is generally too late to administer appropriate treatment (14). Etiology of AMI includes embolic or thrombotic arterial occlusion in 60 to $70 \%$ of the cases, non-occlusive ischemia or infarction in 20 to $30 \%$, and mesenteric venous thrombosis in 5 to $10 \%$. SMA is involved in $85 \%$ of the cases with embolic or thrombotic arterial occlusion (10). Acute and complete cessation of intestinal blood flow leads to perfusion abnormality in the intestinal wall, resulting in the initiation of a process with high mortality in the absence of urgent treatment due to gangrene, ileus, sepsis, and multiorgan failure following bacterial translocation and infiltration after the collapse of mucosal barrier and bacterial translocation (15). Mortality rate with acute treatment after symptom onset is between 0 and $10 \%$, while this figure rises quickly to $50-60 \%$ and $80-100 \%$ with delays of 6-12 hours and $>24$ hours, respectively (16). Despite these high mortality figures, the average delay between symptom onset and establishment of a diagnosis of AMI and initiation of treatment is around 11 hours, showing the need for improved diagnostic performance $(10,17)$. Until now many serum parameters have been tested with regard to their ability to diagnose AMI accurately, and none of these markers have proven sensitive and specific enough to facilitate early diagnosis and to predict early mortality. Similarly, although elevated serum lactate and D-dimer levels are associated with increased mortality in AMI, they are not specific for predicting this increase $(9,18)$.

Ultimately, reduced mortality rate in AMI requires early diagnosis and treatment. Recent studies showed that NLR may represent an important marker for cardiac and non-cardiac conditions, and particularly in detecting the inflammation in malignancies, gastrointestinal conditions, and chronic renal failure (19-22). Toptas et al. (23) reported significantly higher NLR and PLR in patients with AMI as compared to controls where a NLR greater than 4.5 had $77 \%$ sensitivity and $72 \%$ specificity for a diagnosis of AMI and a PLR greater than 157 had 59\% and 65\% sensitivity and specificity, respectively. Aktimur et al. (24) found a sensitivity and specificity of $74.3 \%$ and $82.9 \%$, respectively for a NLR of $\geq 9.9$ in the diagnosis of AMI and they reported even higher diagnostic yield when NLR and RDW were combined. In another study, a NLR level of greater than 4.68 as a marker of inflammation was associated with acute appendicitis, while higher values $(25.74)$ were indicative of complicated appendicitis (25). In the current study comparing NLR and PLR for diagnosis of AMI, it was found that AMI+ patients had significantly higher values than AMI- patients ( $\mathrm{p}<0.001, \mathrm{p}<0.001$, respectively). In contrast with the above-mentioned studies, although ROC analysis on the predictive value of NLR and PLR for inhospital mortality in patients with AMI identified a best cut-off value of $>10.1$ for NLR, no such values could be identified for PLR. In other words, NLR exhibited a significantly higher "area under curve" value. In our participants, the overall inhospital mortality rate was $48 \%$ versus $66 \%$ in those with a NLR of greater than 10.1, suggesting that NLR, as a strong marker of inflammation, could represent a more valuable indicator of inhospital mortality in patients with AMI as compared to PLR. A significantly increased release of neutrophils into circulation occurs in response to inflammation and neutrophils play a significant role in tissue destruction and injury. In contrast with neutrophils, number of circulatory lymphocytes decreases during inflammation due to increased steroid release due to stress and increased apoptotic cell death (26). In our study, patients who died had statistically insignificant increase in their neutrophil count as compared to patients who survived. (13.9 \pm 3.3 , 11.6 $\pm 4.4, p=0.07$, respectively). However, patients, who died had significantly lower lymphocyte count $(1.3 \pm 0.3,3.0 \pm 1.3$, $\mathrm{p}=0.015$, respectively). Also, no significant differences in platelet counts were observed between those patients who did and did not survive (302.2 $\pm 115.4,279.5 \pm 53.7, \mathrm{p}=0.890$, respectively). Interestingly, despite there was a significant initial difference in neutrophil counts between AMI+ and AMI- patients, no such differences could be identified in platelet counts. Among AMI+ patients who died, there was an insignificant increase in neutrophil counts in conjunction with a significant decrease in lymphocytes. Although the cause of the decrease in lymphocyte counts could not be fully elucidated, it may be associated with the increased release of cortisol secondary to the concurrent occurrence of thrombus and ischemia in the mesenteric artery. Based on our observations, we believe that the increase in NLR in our study is associated with the delay in diagnosis, extent of the intestinal segment with high risk of necrosis, and increased neutrophil count and decreased lymphocyte count secondary to the infection and inflammation. While a lower cut-off value for NLR may represent an important parameter for AMI diagnosis, NLR may predict inhospital mortality at higher cut-off levels. Further prospective studies with larger sample size are warranted for defining the role of these novel hematological parameters in the diagnosis of AMI and in predicting its mortality.

Our study has several limitations. Firstly, it is a single-center and retrospective study. Also, the time from presentation to diagnosis and the type of treatment administered were not assessed. Thirdly, the increase in CRP, a non-specific marker of inflammation, in AMI+ patients led to the consideration of increased NLR and PLR values in AMI patients as a strong marker of inflammation. Although it is interesting to note a significant increase of NLR in both patients with diagnosis of AMI and inhospital mortality of AMI+ patients, its association with clinical course could not be 
fully understood. Finally, thrombus formation causing AMI was not confirmed histopathologically.

\section{Conclusion}

In this study, high NLR and PLR were found to have significant value in the diagnosis of AMI, while only elevated NLR was significant for predicting inhospital mortality. We believe that as compared with other inflammatory markers, NLR represents a practical, economical, accessible, and useful parameter that can be utilized particularly in the absence of advanced imaging modalities and experienced radiologist assessment. However, further prospective studies are warranted to ascertain the role of NLR as a reliable marker for predicting inhospital mortality in AMI.

\section{Ethics}

Ethics Committee Approval: Şişli Hamidiye Etfal Training and Research Hospital 07.08.2018/2066.

Informed Consent: It was not taken due to a retrospective study.

Peer-review: Externally peer-reviewed.

\section{Authorship Contributions}

Concept: S.S.Y., M.A., G.A., Design: G.E., H.K., S.S., S.S.Y, Data Collection or Processing: G.E., S.S.Y., H.K., M.A., Analysis or Interpretation: H.K., G.A., M.A., S.S., Literature Search: S.S.Y., G.A., H.K., M.A., Writing: G.A., S.S.Y., H.K., S.S., G.E.

Conflict of Interest: No conflict of interest was declared by the authors.

Financial Disclosure: The authors declared that this study has received no financial support.

\section{References}

1. Menke J. Diagnostic accuracy of multidetector CT in acute mesenteric ischemia: systematic review and meta-analysis. Radiology 2010;256:93-01.

2. Oldenburg WA, Lau LL, Rodenberg TJ, Edmonds HJ, Burger CD. Acute mesenteric ischemia: a clinical review. Arch Intern Med 2004; 164:1054-62.

3. Schoots IG, Koffeman GI, Legemate DA, Levi M, van Gulik TM. Systematic review of survival after acute mesenteric ischaemia according to disease aetiology. Br J Surg 2004;91:17-27.

4. van den Heijkant TC, Aerts BA, Teijink JA, Buurman WA, Luyer MD. Challenges in diagnosing mesenteric ischemia. World J Gastroenterol 2013;19:1338-41.

5. Aktekin A, Emir S, Saglam A. Factors affecting mortality in acute mesenteric obstruction. Ulus Travma Acil Cerrahi Derg 2009;15:21721.

6. Woo K, Major K, Kohanzadeh S, Allins AD. Laparotomy for visceral ischemia and gangrene. Am Surg 2007;73:1006-8.
7. Evennett NJ, Petrov MS, Mittal A, Windsor JA. Systematic review and pooled estimates for the diagnostic accuracy of serological markers for intestinal ischemia. World J Surg 2009;33:1374-83.

8. Oldenburg WA, Lau LL, Rodenberg TJ, Edmonds HJ, Burger CD. Acute mesenteric ischemia: a clinical review. Arch Intern Med 2004;164:1054-62.

9. Akyildiz H, Akcan A, Oztürk A, Sozuer E, Kucuk C, Karahan I. The correlation of the D-dimer test and biphasic computed tomography with mesenteric computed tomography angiography in the diagnosis of acute mesenteric ischemia. Am J Surg 2009;197:429-33.

10. Paladino NC, Inviati A, Di Paola V, Busuito G, Amodio E, Bonventre $S$, et al. Predictive factors of mortality in patients with acute mesenteric ischemia. A retrospective study._Ann Ital Chir 2014;85:265-70.

11. Gawaz M, Langer H, May AE. Platelets in inflammation and atherogenesis. J Clin Invest 2005;115:3378-84.

12. Chen J, Chen MH, Li S, Guo YL, Zhu CG, Xu RX, et al. Usefulness of the neutrophil-to-lymphocyte ratio in predicting the severity of coronary artery disease: a Gensini score assessment. J Atheroscler Thromb 2014;21:1271-82.

13. Kundi H, Balun A, Çiçekcioğlu H, Çetin M, Kızıltunç E, Çetin ZG, et al. Association between platelet to lymphocyte ratio and saphenous vein graft disease in patients with stable angina pectoris. Anatol J Cardiol 2016;16:349-53.

14. Acosta S. Epidemiology of mesenteric vascular disease: clinical implications. Semin Vasc Surg 2010;23:4-8.

15. Klar E, Rahmanian PB, Bücker A, Hauenstein K, Jauch KW, Luther $\mathrm{B}$, et al. Acute mesenteric ischemia: a vascular emergency. Dtsch Arztebl Int 2012;109:249-56.

16. Paes E, Vollmar JF, Hutschenreiter S, Schoenberg MH, Schölzel E. Diagnostik und Therapie des akuten Mesenterial infarktes. Chir Gastroenterol 1990;6:473-80.

17. Dahlke MH, Asshoff L, Popp FC, Feuerbach S, Lang SA, Renner $\mathrm{P}$, et al. Mesenteric ischemia-outcome after surgical therapy in 83 patients. Dig Surg 2008;25:213-9.

18. Wyers MC. Acute mesenteric ischemia: diagnostic approach and surgical treatment. Semin Vasc Surg 2010;23:9-20.

19. Zhao QT, Yang Y, Xu S, Zhang XP, Wang HE, Zhang H, et al. Prognostic role of neutrophil to lymphocyte ratio in lung cancers: a meta-analysis including 7,054 patients. Onco Targets Ther 2015;8:2731-8.

20. Yaylak B, Ede H, Baysal E, Altıntas B, Akyuz S, Sevuk U, et al. Neutrophil/lymphocyte ratio is associated with right ventricular dysfunction in patients with acute inferior ST elevation myocardial infarction._Cardiol J 2016;23:100-6.

21. Malhotra R, Marcelli D, von Gersdorff G, Grassmann A, Schaller M, Bayh I, et al. Relationship of Neutrophil-to-Lymphocyte Ratio and Serum Albumin Levels with C-Reactive Protein in Hemodialysis Patients: Results from 2 International Cohort Studies. Nephron 2015;130:263-70. 
22. Farah R, Khamisy-Farah R. Association of neutrophil to lymphocyte ratio with presence and severity of gastritis due to Helicobacter pylori infection. J Clin Lab Anal 2014;28:219-23.

23. Toptas M, Akkoc İ, Savas Y, Uzman S, Toptas Y, Can MM. Novel hematologic inflammatory parameters to predict acute mesenteric ischemia. Blood Coagul Fibrinolysis 2016;27:127-30.

24. Aktimur R, Cetinkunar S, Yildirim K, Aktimur SH, Ugurlucan M, Ozlem N. Neutrophil-to-lymphocyte ratio as a diagnostic biomarker for the diagnosis of acute mesenteric ischemia. Eur J Trauma Emerg Surg 2016;42:363-8.

25. Kahramanca S, Ozgehan G, Seker D, Gökce EI, Seker G, Tunç G, et al. Neutrophil-to-lymphocyte ratio as a predictor of acute appendicitis. Turk J Trauma Emerg Surg 2014;20:19-22.

26. Tsokos GC, Liossis SN. Lymphocytes, cytokines, inflammation, and immune trafficking. Curr Opin Rheumatol 1998;10:417-25. 